\title{
Classical IL-6 signaling: a promising therapeutic target for pulmonary arterial hypertension
}

\author{
Soni Savai Pullamsetti, Werner Seeger, and Rajkumar Savai \\ Department of Internal Medicine, Universities of Giessen and Marburg Lung Center (UGMLC), member of the German Center for Lung Research (DZL), Justus Liebig University, Giessen, Germany. \\ Max Planck Institute for Heart and Lung Research, Department of Lung Development and Remodeling, member of the DZL, Bad Nauheim, Cermany.
}

\begin{abstract}
Current therapies for pulmonary arterial hypertension (PAH) provide symptomatic relief and improve prognosis but fall short of improving long-term survival. There is emerging evidence for a role of inflammatory mediators, primarily IL-6, in the pathogenesis of PAH. However, the mechanisms by which IL-6 potentially affects PAH are unknown. In this issue of the $J C l$, Tamura, Phan, and colleagues identified ectopic upregulation of the membrane-bound IL-6 receptor (IL6R), indicating classical IL-6 signaling in the smooth muscle layer of remodeled vessels in human and experimental PAH. They performed a series of in vitro and in vivo experiments that provide deeper insights into the mechanisms of classical IL-6 signaling and propose interventions directed against IL6R as a potential therapeutic strategy for PAH.
\end{abstract}

\section{IL-6 signaling in pulmonary hypertension}

IL-6 is a pleiotropic cytokine with a wide range of biologic activities in immune regulation, hematopoiesis, and inflammation, as well as metabolic, proliferative, and regenerative processes. Over the past decade it has become clear that IL-6 utilizes two different pathways to mediate its biological effects: the classical, or cis-signaling, pathway; and the transsignaling pathway. Classical IL-6 receptor signaling denotes activities mediated by membrane-bound IL6R $\alpha$ (referred to herein as IL6R) and therefore is relevant only in cells that express IL6R. Hence, classical IL-6 signaling occurs mainly in the liver and in leukocyte subpopulations. IL-6 binds to IL6R on these cells, which induces the formation of a heterohexameric complex consisting of two molecules each of IL-6, IL6R, and IL-6 receptor subunit $\beta$ (gp130). Formation of this complex results in the activation of the JAK/ STAT3 signaling pathway, leading to the transcription of STAT3 target genes. The
IL-6/IL6R/gp130 complex can also activate other signaling pathways, such as PI3K/AKT and MAPK/ERK (Figure 1). In cells not expressing membrane-bound IL6R, circulating IL-6 initiates transsignaling by binding to soluble IL6R (sIL6R) complexes, which leads to dimerization of the gp130 receptor subunit. Binding to sIL6R increases the circulating half-life of IL-6 and promotes its bioavailability (1). Importantly, each pathway regulates distinct biological effects of IL-6. Classical IL-6 signaling is particularly important for the acute-phase immunological response and promotes antiinflammatory activities, whereas IL-6 trans-signaling promotes proinflammatory activities.

Pulmonary hypertension (PH) is a progressive disease with a multifactorial etiology and a poor prognosis, and results in right-sided heart failure. The vascular pathology of $\mathrm{PH}$ is characterized by pulmonary vasoconstriction and abnormal remodeling processes that can affect all vessel layers (2). Mounting evidence suggests a link between the immune system

Related Article: p. 1956

Conflict of interest: The authors have declared that no conflict of interest exists

Reference information: / Clin Invest. 2018;128(5):1720-1723. https://doi.org/10.1172/JCI120415.

and inflammatory mechanisms in the development of group $1 \mathrm{PH}$, i.e., pulmonary arterial hypertension (PAH) $(3,4)$. Elevated circulating levels and enhanced perivascular expression of proinflammatory cytokines (e.g., IL-6) have repeatedly been observed in PAH patients. Indeed, elevated circulating levels of IL- 6 correlate with right ventricular function, worse clinical outcome, greater incidence of quality of life-related symptoms, and/or poorer survival in PAH patients (5-8). Elevated circulating levels of IL- 6 have been found even in the non-PAH forms of $\mathrm{PH}$, such as chronic obstructive pulmonary disease-related $\mathrm{PH}$ (COPD-PH), and in $\mathrm{PH}$ patients with advanced heart failure (9, 10). Furthermore, single nucleotide polymorphisms in the promoter region of IL-6 $(-572 \mathrm{C} / \mathrm{G})$ have been shown to be associated with increased serum IL-6 levels and risk of PAH and COPD-PH $(10,11)$, suggesting a pathogenic role for IL-6 in PH.

Consistent with these findings, administration of IL-6 ligand or overexpression of lung-specific IL-6 in mice leads to spontaneous development of $\mathrm{PH}$ under normoxic conditions and development of much more severe $\mathrm{PH}$ after exposure to chronic hypoxia $(12,13)$. Conversely, IL-6deficient mice are resistant to hypoxiainduced lung inflammation and pulmonary vascular remodeling (14). Although these findings suggest that IL-6 plays a significant role in the pathogenesis of $\mathrm{PH}$, it remains unclear how this signaling pathway determines the progression and ultimately the severity of the disease.

In this issue of the JCI, Tamura, Phan, and colleagues identified ectopic upregulation of membrane-bound IL6R in the smooth muscle layer of remodeled vessels in human idiopathic PAH (iPAH) and experimental models of $\mathrm{PAH}$, and demonstrated a key role for IL6R in pulmonary arterial smooth muscle cell (PASMC) accumulation in vitro and in vivo (15). Additionally, smooth muscle-specific 
deletion of IL6R protected against chronic hypoxia-induced $\mathrm{PH}$ via reduced PASMC accumulation and inflammatory cell infiltration, revealing the potent pro-survival and inflammation-regulatory effects of membrane-bound IL6R. Importantly, treatment with an IL6R-specific antagonist prevented or reversed experimental $\mathrm{PAH}$ induced by monocrotaline (MCT) or hypoxia plus SU5416 in rat models.

\section{IL-6 classical versus trans-signaling in $\mathrm{PAH}$}

How do these findings help expand our understanding of IL-6/IL6R signaling in $\mathrm{PAH}$ pathogenesis and thereby establish new therapeutic strategies?

The authors should be applauded for their effort in dissecting the specific modulation and contribution of the IL- 6 classical versus trans-signaling pathways in human and experimental models of PAH. Interestingly, they identified ectopic upregulation of IL6R (representing the classical IL-6 signaling pathway) in cells other than leukocyte populations, i.e., the PASMCs of patients with IPAH. In addition, the authors also found that deletion of IL6R in the smooth muscle layer causes the regression of hypoxia-induced PAH (Figure 1). To our knowledge, this is the first study that describes activation of the classical IL-6 signaling pathway in PAH pulmonary vasculature. Studies on IL-6 trans-signaling, on the other hand, conducted by Jasiewicz and colleagues, used the serum concentrations of IL-6 signaling molecules to show that IL-6 trans-signaling is enhanced in patients with PAH (16). This is consistent with previous studies suggesting that IL-6 trans-signaling operates in the cells of the $\mathrm{PAH}$ vascular microenvironment (i.e., endothelial cells, smooth muscle cells, fibroblasts, macrophages, and $\mathrm{T}$ lymphocytes), whereas classical IL-6 signaling is activated only in macrophages and PASMCs under PAH conditions $(17,18)$.

Essential questions that further arise include: What mechanisms underlie the ectopic expression of membrane-bound IL6R in PASMCs in response to pathological stimuli? Are both the classical and trans-signaling pathways activated under these conditions, and what are the consequences of this activation for vascular remodeling? Which of these two IL-6 signaling pathways contributes to progressive vascular remodeling and promotes the development of PAH?

Further investigation is required to identify the downstream signaling molecules that are activated by the classical and/or trans IL-6 pathways and determine how they regulate cellular proliferation, survival, metabolism, and extracellular matrix. Previous studies exploring this aspect observed aberrantly elevated activity of STAT3, one of the major transcription factors regulated by IL-6, in both human and experimental models of PAH $(19,20)$. In addition, Hashimoto-Kataoka et al. showed that IL-21 is a downstream target of the IL-6 signaling that promotes hypoxiainduced $\mathrm{PH}$ by enhancing M2 macrophage polarization (21). Although these studies provide some insight into the few downstream targets of IL-6 signaling pathway, sophisticated and integrative systems biology approaches are warranted to obtain broader insights into the downstream signaling pathways and molecules regulated by IL-6 classical and trans-signaling.

\section{Therapeutic strategies for the blockade of IL-6/IL6R/gp130/ STAT3 signaling in PAH}

Various targeted agents have been developed to inhibit different nodes of the IL-6 signaling pathways: (a) directly targeting IL-6; (b) targeting IL6R to inhibit both the classical and trans-signaling pathways; (c) targeting the IL-6-sIL6R complex using gp130 fusion proteins to selectively inhibit trans-signaling; and small molecule inhibitors targeting (d) JAK phosphorylation or (e) STAT3 function/expression (ref. 22 and Figure 1).

Tamura, Phan, and colleagues studied the efficacy of the nonpeptide IL6R/sIL6R antagonist 20S,21-epoxy-resibufogenin3-formate (ERBF) in MCT- and hypoxia plus SU5416-induced PAH. Interestingly, daily treatment with ERBF prevented or reversed PAH (15). Likewise, a gp130 inhibitor, SC144, ameliorated pulmonary vascular remodeling in MCT-induced $\mathrm{PAH}$ in rats (23). These two studies suggest that simultaneous inhibition of IL-6 classical and trans-signaling pathways may hold promise for future clinical studies in patients with PAH. Furthermore, recent data demonstrate that STAT3 can be therapeutically targeted in different animal models of PAH (19).
Importantly, tocilizumab (currently approved for the treatment of rheumatoid arthritis), a humanized monoclonal antibody that recognizes IL6R and disrupts both classical and trans-signaling, improved $\mathrm{PAH}$ symptoms in a patient with mixed connective tissue disease and severe PAH (24). To build upon this result, an open-label study has been launched to assess the efficacy and safety of tocilizumab in PAH patients (ClinicalTrials. gov NCT02676947). The toxicity profile of tocilizumab is manageable but includes infection, neutropenia, and elevated cholesterol levels (25).

\section{Challenges in the translation of IL6R-blocking therapies in $\mathrm{PAH}$ patients}

Blocking all IL-6 signaling might have severe consequences because IL-6 serves many vital functions. Thus, developing a method for selective blockade of the pathogenic signals of IL- 6 while leaving certain IL-6-regulated homeostatic processes intact is warranted. The study by Tamura, Phan, and colleagues suggests that blocking classical IL-6 signaling specifically via IL6R blockade could be therapeutically relevant for patients with $\mathrm{PAH}$. However, ERBF, the agent used for treatment in preclinical models of $\mathrm{PAH}$, cannot differentiate between IL-6 classical and trans-signaling (15). Thus, an emerging challenge is to discover how to specifically target the proinflammatory trans pathway. This will help determine whether such therapy offers a clinical advantage over global inhibition of either IL6R or IL-6.

It remains unknown whether the same molecular mechanisms studied in the context of iPAH apply to other subgroups of patients with $\mathrm{PAH}$ or $\mathrm{PH}$. Although elevated levels of IL- 6 are observed in several groups of $\mathrm{PH}$, it is not known whether this exerts a pathogenic or protective role in vascular remodeling in those $\mathrm{PH}$ patients $(9,10,26)$.

Although not specifically discussed here, there is clear evidence that the response of the right ventricle to pressure (i.e., afterload) varies by sex, age, and likely many other genetic and environmental factors. Another important consideration is whether these putative IL6R therapies, aimed at specifically targeting abnormal signaling in the pulmonary vasculature, also normalize right ventricular function. 


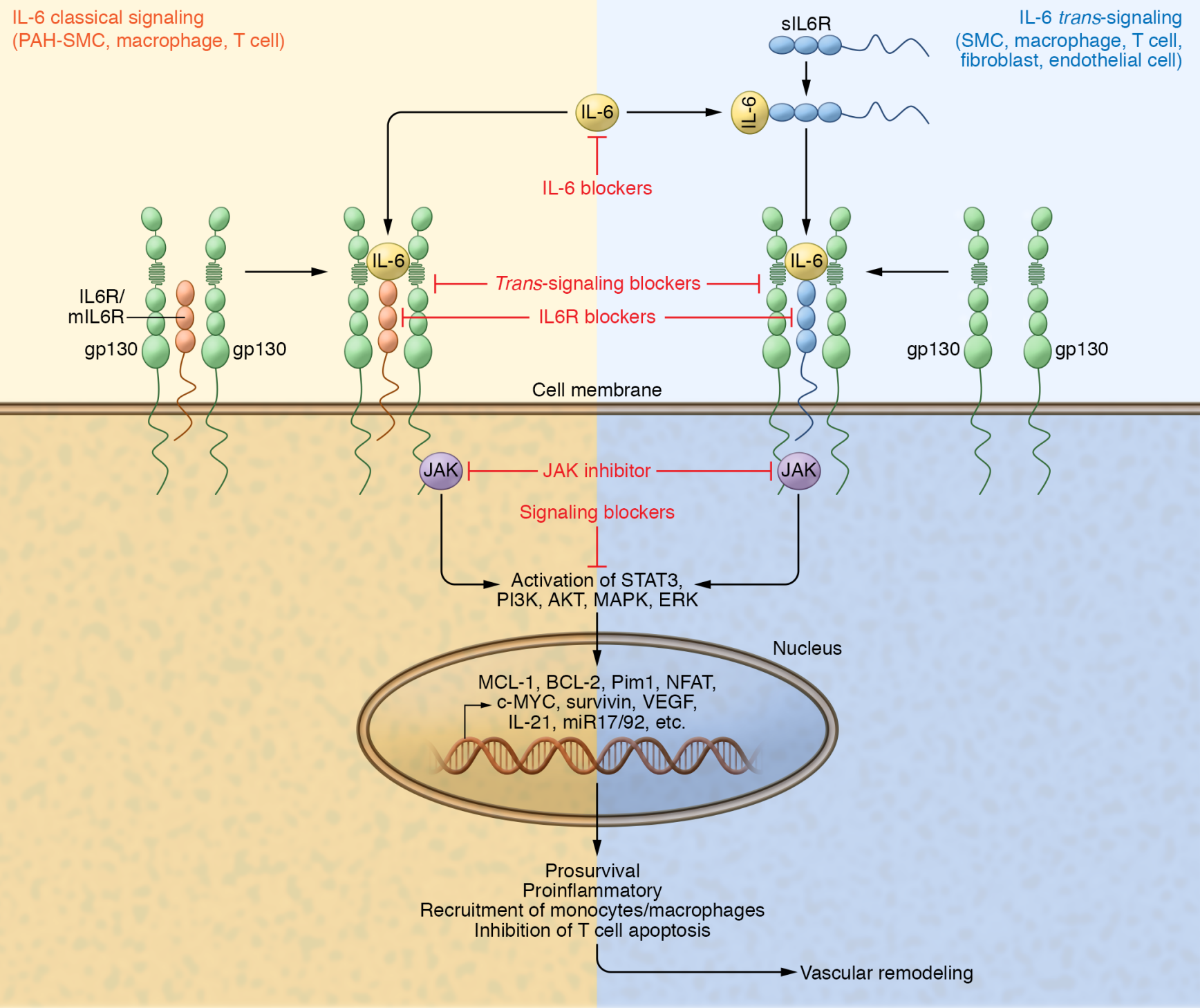

Figure 1. IL-6 classical and trans-signaling in PAH. In classical signaling, IL-6 binds to its membrane-bound receptor (IL6R/mIL6R), inducing the formation of a heterohexameric complex containing two molecules each of IL-6, IL6R, and the IL-6 receptor subunit $\beta$ (gp130). Formation of this complex results in activation of the JAK/STAT3 signaling pathway, leading to the transcription of STAT3 target genes. The IL-6/IL6R/gp130 complex can also activate other signaling pathways, such as PI3K/AKT and MARK/ERK. In trans-signaling, IL-6 binds to the soluble form of the IL-6 receptor (sIL6R) and forms a binary complex that can bind to any cell that expresses gp130 on its surface. Through these signaling pathways, IL-6 exerts its pro-survival and proinflammatory recruitment of mononuclear cells and inhibition of T cell apoptosis. Pulmonary IL-6 production is increased in PAH patients and is thought to reflect increased production by both inflammatory cells and vascular cells. IL6R is expressed in a limited number of cell types under normal physiological conditions, including mainly immune/inflammatory cells (e.g., macrophages and T lymphocytes), whereas IL6R is ectopically upregulated in pulmonary arterial smooth muscle cells (SMC) in PAH patients and models. Because gp130 is ubiquitously expressed, trans-signaling can occur upon IL-6 stimulation in all vascular and non-vascular cells. Therapeutic strategies for the blockade of different nodes of the IL-6 signaling pathway (shown in red) have been developed.

Finally, it is important to identify the patients who may benefit most from IL6R-blocking therapies. Many of the diseases in which inhibition of IL-6 is clinically beneficial are associated with dysregulated adaptive immunity (27). Thus, it is important to identify biomarkers to predict therapeutic responses to IL6R blockers in patients with PAH by employing pharmacogenomics, kinomics, phosphoproteomics, immunophenomics, and RNA-sequencing technologies.

\section{Concluding remarks}

In summary, interventions directed against IL6R/gp130 signaling represent excellent potential therapies for PAH. However, as evidenced by a series of in vitro and in vivo experiments by Tamura, Phan, and colleagues, we need to identify the contri- butions of IL-6 classical and trans-signaling and the therapeutic responses to their selective blockade in PAH (15). This field of research is likely to broaden over the coming years. The emerging challenge is to determine how to specifically target IL-6 pathways and how to identify the patients who would benefit most from IL6R-blocking therapies. Importantly, considering the long-standing efforts of the author's 
group toward addressing inflammation and altered immunity in PAH as a therapeutic strategy, we can envisage clinical translation of IL6R-blocking therapies in the near future.

\section{Acknowledgments}

This work was supported by the Max Planck Society, Excellence Cluster Cardio-Pulmonary System, German Center for Lung Research (DZL), and SFB 1213 project A01.

Address correspondence to: Soni Savai Pullamsetti, Molecular Mechanisms of Pulmonary Vascular Diseases, Department of Lung Development and Remodeling, Max Planck Institute for Heart and Lung Research, Parkstrasse 1, D-61231 Bad Nauheim, Germany. Phone: 49.6032. 705.380; Email: soni.pullamsetti@mpi-bn. mpg.de.

1. Hunter CA, Jones SA. IL-6 as a keystone cytokine in health and disease. Nat Immunol. 2015;16(5):448-457.

2. Rabinovitch M. Molecular pathogenesis of pulmonary arterial hypertension. J Clin Invest. 2012;122(12):4306-4313.

3. Dorfmüller P, Perros F, Balabanian K, Humbert M. Inflammation in pulmonary arterial hypertension. Eur Respir J. 2003;22(2):358-363.

4. Savai R, et al. Immune and inflammatory cell involvement in the pathology of idiopathic pulmonary arterial hypertension. Am J Respir Crit Care Med. 2012;186(9):897-908.

5. Cracowski JL, et al. Proinflammatory cytokine levels are linked to death in pulmonary arterial hypertension. Eur Respir J. 2014;43(3):915-917.

6. Humbert $M$, et al. Increased interleukin-1 and interleukin-6 serum concentrations in severe primary pulmonary hypertension. Am J Respir
Crit Care Med.1995;151(5):1628-1631.

7. Soon E, et al. Elevated levels of inflammatory cytokines predict survival in idiopathic and familial pulmonary arterial hypertension. Circulation. 2010;122(9):920-927.

8. Prins KW, et al. Interleukin- 6 is independently associated with right ventricular function in pulmonary arterial hypertension. J Heart Lung Transplant. 2018;37(3):376-384

9. Dolenc J, Šebeštjen M, Vrtovec B, Koželj M, Haddad F. Pulmonary hypertension in patients with advanced heart failure is associated with increased levels of interleukin-6. Biomarkers. 2014;19(5):385-390.

10. Chaouat A, et al. Role for interleukin- 6 in COPD-related pulmonary hypertension. Chest. 2009;136(3):678-687.

11. Fang M, Huang Y, Zhang Y, Ning Z, Zhu L, Li X. Interleukin-6 $-572 \mathrm{C} / \mathrm{G}$ polymorphism is associated with serum interleukin-6 levels and risk of idiopathic pulmonary arterial hypertension. JAm Soc Hypertens. 2017;11(3):171-177.

12. Golembeski SM, West J, Tada Y, Fagan KA. Interleukin- 6 causes mild pulmonary hypertension and augments hypoxia-induced pulmonary hypertension in mice. Chest. 2005; 128(6 suppl):572S-573S.

13. Steiner MK, Syrkina OL, Kolliputi N, Mark EJ, Hales CA, Waxman AB. Interleukin- 6 overexpression induces pulmonary hypertension. Circ Res. 2009;104(2):236-244.

14. Savale L, et al. Impact of interleukin- 6 on hypoxiainduced pulmonary hypertension and lung inflammation in mice. Respir Res. 2009;10:6.

15. Tamura Y, et al. Ectopic upregulation of membrane-bound IL6R drives vascular remodeling in pulmonary arterial hypertension. JClin Invest. 2018;128(5):1956-1970.

16. Jasiewicz M, et al. Enhanced IL-6 trans-signaling in pulmonary arterial hypertension and its potential role in disease-related systemic damage. Cytokine. 2015;76(2):187-192.

17. Le Hiress $\mathrm{M}$, et al. Proinflammatory signature of the dysfunctional endothelium in pulmonary hypertension. Role of the macrophage migration inhibitory factor/CD74 complex. Am J Respir Crit Care Med. 2015;192(8):983-997.
18. El Kasmi KC, et al. Adventitial fibroblasts induce a distinct proinflammatory/profibrotic macrophage phenotype in pulmonary hypertension. J Immunol. 2014;193(2):597-609.

19. Paulin R, et al. Signal transducers and activators of transcription-3/pim1 axis plays a critical role in the pathogenesis of human pulmonary arterial hypertension. Circulation. 2011;123(11):1205-1215.

20. Brock M, et al. Interleukin- 6 modulates the expression of the bone morphogenic protein receptor type II through a novel STAT3microRNA cluster 17/92 pathway. Circ Res. 2009;104(10):1184-1191

21. Hashimoto-Kataoka T, et al. Interleukin-6/interleukin-21 signaling axis is critical in the pathogenesis of pulmonary arterial hypertension. Proc Natl Acad Sci U S A . 2015;112(20):E2677-E2686.

22. Johnson DE, O'Keefe RA, Grandis JR. Targeting the IL-6/JAK/STAT3 signalling axis in cancer [published online ahead of print Feburary 6, 2018]. Nat Rev Clin Oncol. https://doi. org/10.1038/nrclinonc.2018.8.

23. Huang Z, et al. Glycoprotein 130 inhibitor ameliorates monocrotaline-induced pulmonary hypertension in rats. Can J Cardiol. 2016;32(11):1356.e1-1356.e10.

24. Furuya Y, Satoh T, Kuwana M. Interleukin-6 as a potential therapeutic target for pulmonary arterial hypertension. Int J Rheumatol. 2010;2010:720305.

25. Rubbert-Roth A, Furst DE, Nebesky JM, Jin A, Berber E. A review of recent advances using tocilizumab in the treatment of rheumatic diseases [published online ahead of print March 3 2018]. Rheumatol Ther. https://doi.org/10.1007/ s40744-018-0102-x.

26. Graham BB, et al. Protective role of IL-6 in vascular remodeling in Schistosoma pulmonary hypertension. Am J Respir Cell Mol Biol. 2013;49(6):951-959.

27. Sanayama Y, et al. Prediction of therapeutic responses to tocilizumab in patients with rheumatoid arthritis: biomarkers identified by analysis of gene expression in peripheral blood mononuclear cells using genomewide DNA microarray. Arthritis Rheumatol. 2014;66(6):1421-1431. 